\title{
Notification that New Names and New Combinations Have Appeared in Volume 45, No. 1, of the IJSB $^{a}$
}

\begin{tabular}{|c|c|c|c|}
\hline Name: & Proposed as: & Authors: & $\begin{array}{l}\text { IJSB reference } \\
\text { description: }\end{array}$ \\
\hline $\begin{array}{l}\text { Bartonella } \\
\text { Bartonella talpae (basonym Grahamella talpae) } \\
\text { Bartonella peromysi (basonym Grahamella peromysci) } \\
\text { Bartonella grahamii } \\
\text { Bartonella taylorii } \\
\text { Bartonella doshiae } \\
\text { Bacillus thermoamylovorans } \\
\text { Microlunatus } \\
\text { Microlunatus phosphovorus } \\
\text { Spiroplasma ixodetis } \\
\text { Mycoplasma adleri } \\
\text { Dietzia } \\
\text { Dietzia maris (basonym Rhodococcus maris) } \\
\text { Bordetella hinzii } \\
\text { Dermatophilus chelonae } \\
\text { Actinomyces bernardiae } \\
\text { Campylobacter hyoilei } \\
\text { Thermocrispum } \\
\text { Thermocrispum municipale } \\
\text { Thermocrispum agreste } \\
\text { Brevinema } \\
\text { Brevinema andersonii } \\
\text { Desulfacinum } \\
\text { Desulfacinum infernum } \\
\text { Porphyromonas salivosa pro synon., Bacteroides macacae } \\
\text { Porphyromonas macacae (basonym Bacteroides macacae) } \\
\text { Bacillus reuszeri } \\
\text { Bacillus formosus } \\
\text { Bacillus borstelensis } \\
\text { Nocardia calcarea pro synon., Rhodococcus erythropolis } \\
\text { Nocardia restricta pro synon., Rhodococcus equi } \\
\text { Rhodococcus roseus pro synon., Rhodococcus rhodochrous } \\
\text { Cycloclasticus } \\
\text { Cycloclasticus pugetii } \\
\text { Amycolatopsis coloradensis } \\
\text { Corynebacterium macginleyi } \\
\text { Vibrio penaeicida } \\
\text { Photobacterium damsela subsp. piscicida (basonym “Pasteurella piscicida") } \\
\text { Photobacterium damsela subsp. damsela } \\
\text { Campylobacter gracilis (basonym Bacteroides gracilis) } \\
\text { Rhizobium tianshanense } \\
\text { Brachybacterium conglomeratum } \\
\text { Brachybacterium paraconglomeratum } \\
\text { Brachybacterium rhamnosum } \\
\text { Staphylococcus pulvereri } \\
\text { Methylomicrobium } \\
\text { Methylomicrobium agile (basonym Methylobacter agilis) } \\
\text { Methylomicrobium album (basonym Methylobacter albus) } \\
\text { Methylomicrobium pelagicum (basonym Methylobacter pelagicus) } \\
\text { Methylobacter }\end{array}$ & $\begin{array}{l}\text { emend. } \\
\text { comb. nov. } \\
\text { comb. nov. } \\
\text { sp. nov. } \\
\text { sp. nov. } \\
\text { sp. nov. } \\
\text { sp. nov. } \\
\text { gen. nov. } \\
\text { sp. nov. } \\
\text { sp. nov. } \\
\text { sp. nov. } \\
\text { gen. nov. } \\
\text { comb. nov. } \\
\text { sp. nov. } \\
\text { sp. nov. } \\
\text { sp. nov. } \\
\text { sp. nov. } \\
\text { gen. nov. } \\
\text { sp. nov. } \\
\text { sp. nov. } \\
\text { gen. nov. } \\
\text { sp. nov. } \\
\text { gen. nov. } \\
\text { sp. nov. } \\
\text { synon. } \\
\text { comb. nov. } \\
\text { sp. nov. } \\
\text { sp. nov., nom. rev. } \\
\text { sp. nov., nom. rev. } \\
\text { synon. } \\
\text { synon. } \\
\text { synon., emend. } \\
\text { gen. nov. } \\
\text { sp. nov. } \\
\text { sp. nov. } \\
\text { sp. nov. } \\
\text { sp. nov. } \\
\text { comb. nov. } b \\
\text { Rule } 46^{c} \\
\text { comb. nov. } \\
\text { sp. nov. } \\
\text { sp. nov., nom. rev. } \\
\text { sp. nov. } \\
\text { sp. nov. } \\
\text { sp. nov. } \\
\text { gen. nov. } \\
\text { comb. nov. } \\
\text { comb. nov. } \\
\text { comb. nov. } \\
\text { emend. } \\
\text { ing }\end{array}$ & 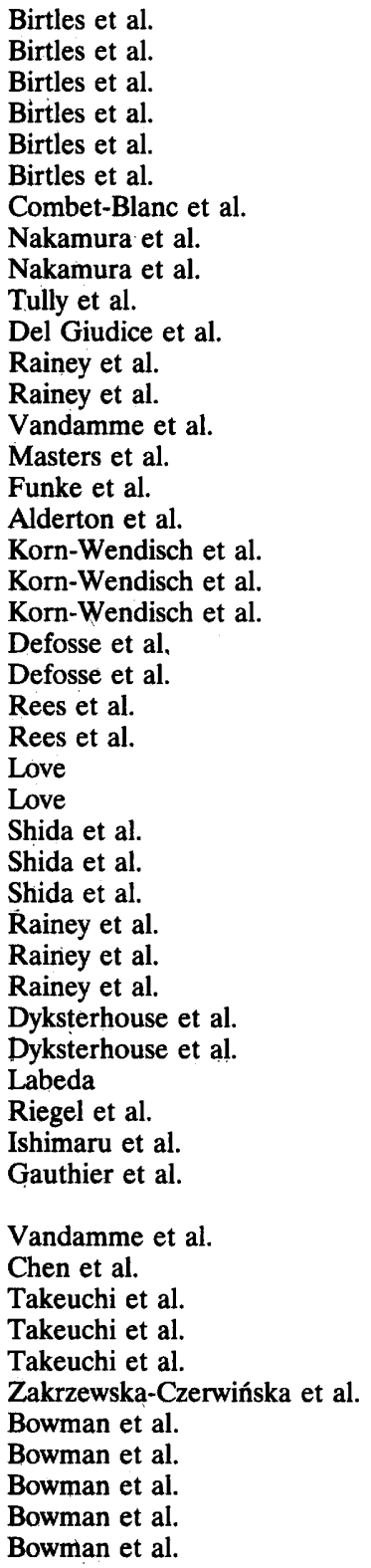 & $\begin{array}{l}\mathbf{4 5}(1): 7 \\
\mathbf{4 5}(1): 7 \\
\mathbf{4 5}(1): 7 \\
\mathbf{4 5}(1): 7 \\
\mathbf{4 5}(1): 7 \\
\mathbf{4 5}(1): 7 \\
\mathbf{4 5}(1): 15 \\
\mathbf{4 5}(1): 21 \\
\mathbf{4 5}(1): 21 \\
\mathbf{4 5}(1): 27 \\
\mathbf{4 5}(1): 31 \\
\mathbf{4 5}(1): 33 \\
\mathbf{4 5}(1): 35 \\
\mathbf{4 5}(1): 43 \\
\mathbf{4 5}(1): 55 \\
\mathbf{4 5}(1): 59 \\
\mathbf{4 5}(1): 65 \\
\mathbf{4 5}(1): 73 \\
\mathbf{4 5}(1): 74 \\
\mathbf{4 5}(1): 75 \\
\mathbf{4 5}(1): 83 \\
\mathbf{4 5}(1): 83 \\
\mathbf{4 5}(1): 88 \\
\mathbf{4 5}(1): 88 \\
\mathbf{4 5}(1): 91 \\
\mathbf{4 5}(1): 91 \\
\mathbf{4 5}(1): 98 \\
\mathbf{4 5}(1): 98 \\
\mathbf{4 5}(1): 98 \\
\mathbf{4 5}(1): 102 \\
\mathbf{4 5}(1): 102 \\
\mathbf{4 5}(1): 102 \\
\mathbf{4 5}(1): 120 \\
\mathbf{4 5}(1): 120 \\
\mathbf{4 5}(1): 126 \\
\mathbf{4 5}(1): 132 \\
\mathbf{4 5}(1): 138 \\
\mathbf{4 5}(1): 142\end{array}$ \\
\hline
\end{tabular}

${ }^{a}$ This listing of names published in a previous issue of IJSB is provided as a service to bacteriology to assist in the recognition of new names and new descriptions. This procedure was proposed by the Judicial Commission [Minute II (ii), Int. J. Syst. Bacteriol. 41:185, 1991]. The names given herein have priority according to the issue of the IJSB in which they were published.

${ }^{b}$ The complete proposal should read: subsp. nov., comb. nov., nom. rev.

${ }^{c}$ According to Rule 46 of the International Code of Nomenclature of Bacteria, the valid publication of Photobacterium damsela subsp. piscicida automatically creates another subspecies, Photobacterium damsela subsp. damsela (Love et al. 1982) Smith et al. 1991. 